\title{
Efficient finite element modeling of radiation forces on elastic particles of arbitrary size and geometry
}

\author{
Peter Glynne-Jones, ${ }^{\text {a) }}$ Puja P. Mishra, Rosemary J. Boltryk, and Martyn Hill \\ Engineering Sciences, Faculty of Engineering and the Environment, University of Southampton, \\ Southampton, SO17 1BJ, United Kingdom
}

(Received 14 June 2011; revised 2 November 2012; accepted 12 February 2013)

\begin{abstract}
A finite element based method is presented for calculating the acoustic radiation force on arbitrarily shaped elastic and fluid particles. Importantly for future applications, this development will permit the modeling of acoustic forces on complex structures such as biological cells, and the interactions between them and other bodies. The model is based on a non-viscous approximation, allowing the results from an efficient, numerical, linear scattering model to provide the basis for the secondorder forces. Simulation times are of the order of a few seconds for an axi-symmetric structure. The model is verified against a range of existing analytical solutions (typical accuracy better than $0.1 \%$ ), including those for cylinders, elastic spheres that are of significant size compared to the acoustic wavelength, and spheroidal particles. (C) 2013 Acoustical Society of America.
\end{abstract}

[http://dx.doi.org/10.1121/1.4794393]

PACS number(s): 43.25.Qp [JDM]

Pages: $1885-1893$

\section{INTRODUCTION}

Ultrasonic standing wave fields can be used to exert a radiation force on small particles, including biological cells. The radiation force is a nonlinear phenomenon generated by the interaction of ultrasound scattered by the particle and energy gradients within the ultrasonic field. The length scales of ultrasonic standing waves are highly compatible with the scales required for manipulation of cells within micro total analysis systems ( $\mu \mathrm{TAS}$ ) and this has led to significant recent interest in the phenomenon. Biological applications of the technology are particularly attractive as there is clear evidence that the acoustic energy required for the levitation and manipulation of cells does not significantly impair their viability. ${ }^{1,2}$

Ultrasonic manipulation is complementary to techniques such as optical trapping and dielectrophoresis as the potential wells generated can be relatively large, making ultrasound suitable for the formation and manipulation of cell agglomerates, but less suitable for the precise manipulation of individual cells.

Recently reported applications of the technology include trapping of cells for microscopy ${ }^{3}$ biosensor enhancement, ${ }^{4}$ fractionation, ${ }^{5}$ medium exchange, ${ }^{6}$ and tissue engineering. ${ }^{7}$ Most of the modeling of radiation forces that underpins these applications has been based on the work of $\mathrm{King}^{8}$ or of Yosioka and Kawasima. ${ }^{9}$ As investigators explore the potential of the technology in biosciences applications, there is a need for models of radiation force that offer more flexibility in terms of the geometry, size and material characteristics of the cells, particles and agglomerates formed within ultrasonic fields. The approach taken in this work uses a finite element representation that allows for the estimation of forces on fluid or elastic scatterers of arbitrary sizes and

\footnotetext{
a) Author to whom correspondence should be addressed. Electronic mail: p.glynne-jones@soton.ac.uk
}

geometries, and with material inhomogeneities. Despite its power and flexibility the implementation is efficient enough to allow multiple parametric studies to be undertaken in acceptable timescales.

\section{BACKGROUND}

The first comprehensive theoretical investigation of radiation forces on small scatterers was undertaken by King in $1934 .{ }^{9}$ King considered a rigid sphere in an inviscid fluid in both plane progressive and plane standing waves. Many authors have subsequently revisited the radiation force problem to overcome one or more of these assumptions. The behavior of bubbles in acoustic fields led Yosioka and Kawasima ${ }^{9}$ to derive an expression for the force on a compressible particle within a plane wave. This was reformulated by Gor'kov ${ }^{10}$ to express the time averaged radiation force, $F(\boldsymbol{r})$ on a sphere of volume $V$ located at $r$ within stationary acoustic fields other than plane standing waves, characterized by their time averaged kinetic and potential energy densities ( $E_{\mathrm{kin}}$ and $E_{\mathrm{pot}}$, respectively):

$$
F(\boldsymbol{r})=\nabla V\left[\frac{3\left(\rho_{p}-\rho_{f}\right)}{\left(2 \rho_{p}+\rho_{f}\right)} E_{\mathrm{kin}}(\boldsymbol{r})-\left(1-\frac{\beta_{p}}{\beta_{f}}\right) E_{\mathrm{pot}}(\boldsymbol{r})\right] .
$$

The energy density terms are weighted by functions of the compressibilities $\left(\beta_{p}\right.$ and $\left.\beta_{f}\right)$ and densities $\left(\rho_{p}\right.$ and $\left.\rho_{f}\right)$ of the particle and the surrounding fluid.

Other authors have studied the phenomenon in specific fields of non-planar geometry. ${ }^{11-13}$ Westervelt ${ }^{14}$ included viscosity effects in radiation force calculations and Doinikov ${ }^{15,16}$ demonstrated that thermal and viscous effects play only a small role on acoustic radiation forces on particles significantly larger than the viscous and thermal penetration depths within standing waves. King ${ }^{17}$ also modeled the force on thin discs in planar fields and subsequent 
investigations have included theory for the forces on discs, ${ }^{18}$ deformed water drops, ${ }^{19}$ cylinders, ${ }^{20-23}$ and ellipsoids. ${ }^{24}$

The theory of Yosioka and Kawasima ${ }^{9}$ was restricted to the force on compressible fluid particles significantly smaller than a wavelength. Hasegawa and Yosioka ${ }^{25}$ and Hasegawa ${ }^{26}$ investigated elastic scatterers (i.e., scatterers that can support shear wave propagation in addition to the purely compressive waves in a fluid scatterer) in plane progressive and stationary waves, and included investigations of particles sizes that were significantly larger than is the case for the small scatterer approximation.

Many studies of the behavior of particles within standing wave fields have relied on the small, compressible fluid scatterer approximations of Yosioka and Kawasima ${ }^{9}$ and

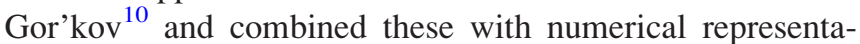
tions of the field itself. Gröschl ${ }^{27}$ used Nowotny's onedimensional transfer matrix model ${ }^{28}$ to predict forces on particles within multi-wavelength resonators and a similar approach by Hill et al. ${ }^{29}$ has been used to predict particle behavior in sub-wavelength planar resonators. ${ }^{30,31}$ Such onedimensional approximations can provide useful information for the design of resonators but fail to represent the complexity of behavior in the lateral directions that is observed in real systems. A number of authors have addressed this using numerically derived two-dimensional representations of acoustic fields ${ }^{32-34}$ which can be combined with Gor'kov's force potential formulation. ${ }^{35,36}$

Other authors have used a numerical formulation to simulate both the field and the radiation force itself, without resorting to Yosioka and Kawasima's approximations. Haydock $^{21}$ used a Lattice Boltzmann approach that included viscosity to calculate the radiation force on cylinders in a standing wave, and Wang and Dual ${ }^{23}$ also used a full numerical simulation of the viscous Navier-Stokes equations to calculate forces on a rigid cylinder, in this case using a finite volume representation. While these simulations are potentially very powerful, particularly in cases such as particles near boundaries where viscosity effects are important, the simulations are typically very computationally demanding.

Cai et $a l .{ }^{20}$ used a finite difference time domain method to calculate the radiation force generated by propagating waves in an inviscid fluid. Again, the computational demand of this method is significant. A finite element approach has been used by Liu et al. ${ }^{37}$ to examine the shape dependency of radiation forces in complex acoustic fields. This has generated useful results relating to the trapping of particles of different shapes, but the analysis is based on a fixed particle boundary so requires assumptions of a rigid, fixed particle.

The work described in this paper combines the efficiency and flexibility (in terms of the geometry of the scatterer and the field) of the finite element method, but uses the approach developed by Yosioka and Kawasima ${ }^{9}$ to allow for the compressibility of the particle, allowing the behavior of both compressible and elastic particles in an inviscid fluid to be modeled. Finally, to validate key aspects of the model and its development, the paper draws from a range of analytical solutions given in the literature including those for cylinders, spheres, and spheroids.

\section{METHOD}

\section{A. Theoretical basis}

To find the acoustic radiation force on an arbitrary shaped particle, we take advantage of the useful relation that in the inviscid approximation, the acoustic radiation forces to second order can be expressed as functions of first-order acoustic quantities. Thus, we can take the results of a computationally efficient, numerical, linear, first-order acoustic scattering simulation and use them to calculate the radiation force on a particle. We will refer to this method as the perturbation FEA method in the remainder of the paper. This approach is very similar to the analytical approach used by Yosioka and Kawasima ${ }^{9}$ to derive the force on a compressible sphere.

In the manner of a perturbation analysis, we write the total pressure and velocity as a series, beginning with the constant steady state terms, followed by the much smaller periodic linear acoustic terms $p_{1}$ and $\boldsymbol{v}_{1}$, followed by the second-order terms $p_{2}$ and $v_{2}$ that are functions of the lowerorder terms.

$$
\begin{aligned}
& p=p_{0}+p_{1}+p_{2}+\cdots, \\
& v=0+v_{1}+v_{2}+\cdots .
\end{aligned}
$$

Bruus $^{38}$ shows that with the approximation of an inviscid fluid, the time averaged second-order acoustic pressure term is given by

$$
\left\langle p_{2}\right\rangle=\frac{1}{2 \rho_{0} c_{a}^{2}}\left\langle p_{1}{ }^{2}\right\rangle-\frac{1}{2} \rho_{0}\left\langle v_{1}{ }^{2}\right\rangle
$$

where $\rho_{0}$ is the quiescent fluid density, and $c_{a}$ is the speed of sound in the medium. This equation is central to this paper, and all the FEA results we present subsequently are based on substituting the first-order quantities derived from a finite element scattering simulation into the equation to obtain the time averaged second-order pressure.

We take the first-order terms to be harmonic, so that to first order the pressure at any point averages to zero. The second-order pressure terms, however, do not average to zero as they are formed from squares of the first-order quantities. If the particle boundary is fixed, with a slip condition at the boundary, the time averaged radiation force on the particle is found by integrating the normal component of the total pressure over the surface of the particle (this is the approach used by $\mathrm{Liu}^{37}$ ). To second order, this is given by

$$
-\boldsymbol{F}_{\text {fixed }}=\left\langle\iint_{S} p_{2} \boldsymbol{n} \mathrm{d} S\right\rangle
$$

where $S$ is the surface of the particle and $\boldsymbol{n}$ the surface normal unit vector.

If the particle is free to move in response to the movements of the fluid, then it is necessary to apply this integration over the moving particle surface, $S(t)$. Performing this integration is difficult to implement. Yosioka and Kawasima ${ }^{9}$ showed that this integral can be approximated (to second order), as the integral of the second-order pressure 


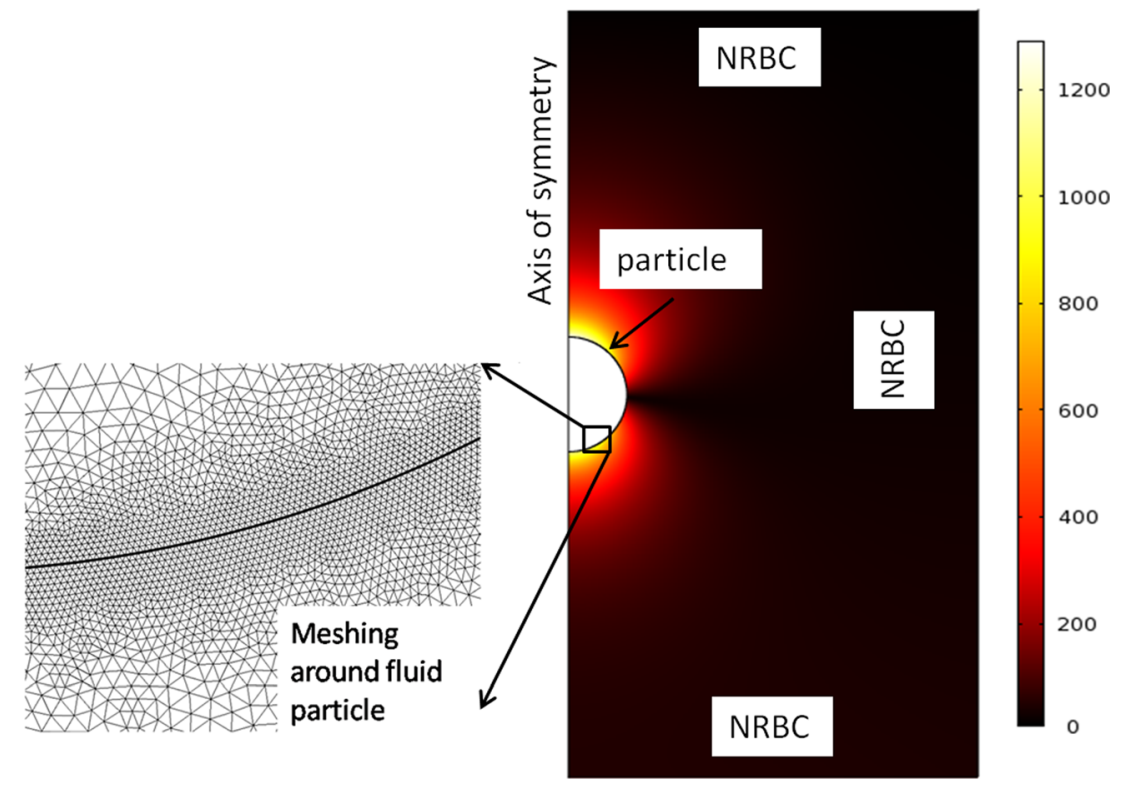

FIG. 1. (Color online) Finite element model to find radiation forces on a compressible sphere along with the scattered pressure distribution $(\mathrm{Pa})$. Nonreflecting boundary conditions marked NRBC. The standing wave field is introduced by specifying acoustic pressures at all of the NRBC boundaries.

component over the equilibrium position of the particle, $S_{0}$, plus a correction term, also integrated over the equilibrium position.

$$
\begin{aligned}
-\boldsymbol{F}_{\text {fixed }}= & \left\langle\iint_{S(t)} p_{2} \boldsymbol{n} \mathrm{dS}\right\rangle=\left\langle\iint_{S_{0}} p_{2} \boldsymbol{n} \mathrm{d} \mathrm{S}\right\rangle \\
& +\left\langle\iint_{S_{0}} \rho_{0}\left(\boldsymbol{n} \cdot \boldsymbol{u}_{1}\right) \boldsymbol{u}_{1} \mathrm{~d} \mathrm{~S}\right\rangle+\cdots
\end{aligned}
$$

where $F$ is the time averaged force on the particle.

The derivation by Gor'kov ${ }^{10}$ for the force on a sphere in an arbitrary field, evaluates the acceleration of the material inside a control surface larger than the particle. The terms include both pressure terms on the boundary, and momentum flux carried across the boundary by fluid crossing the boundary. If the control surface is reduced to the same size as the particle, we find the force takes the same form as Eq. (5) above. Thus Yosioka and Kawasima's correction term for the moving particle can be thought of as a momentum flux through a fixed control surface. In the light of Gor'kov's approach we see that the boundary of integration for Eq. (5) can be a region larger than the particle. It has been verified that the results presented in Secs. IV B and IV C below are insensitive to taking a larger integration boundary.

If the first-order pressure and velocity fields are decomposed into first-order incident and scattered fields, then the squared terms in Eq. (3) can be written out as products, for example,

$$
p^{2}=p_{i}^{2}+2 p_{i} p_{s c}+p_{s c}^{2}
$$

where the subscripts $i, s c$, refer to incident and scattered. The $p_{i}^{2}$ and $v_{i}^{2}$ terms ultimately cancel when integrated over the control surface in Eq. (5) (or lead to a hydrostatic overpressure, as described by Lighthill ${ }^{39}$ ). For a small scattering particle, the $p_{s c}^{2}$ and $v_{s c}^{2}$ terms will also be negligible compared to the larger $p_{i} p_{s c}$ and $\boldsymbol{v}_{i} \boldsymbol{v}_{s c}$ mixed products. These simplifications are implemented in the approximations used by Gor'kov and by Yosioka and Kawasima in their derivations of the forces on a small particles. Numerically, for small particles, it may be more accurate to use these approximations, as the incident squared terms (which should integrate to zero over the particle surface but may not integrate due to mesh discretization) will introduce numerical errors due to their larger magnitude compared to the remaining terms. The squares of the scattered pressure and velocity terms must be included when calculating the forces on scatterers that are a significant size in comparison with a wavelength (see below). Unless otherwise stated (when the approximation is called the "small scattering approximation" and the result is referred to as "mixed terms only"), the total field is used for the calculations in this paper.

\section{B. Numerical implementation}

Figure 1 shows a typical 2D implementation of the model. The model is constructed in COMSOL v.4.0a, a commercial multi-physics finite element method package. In order to establish good agreement with the analytical solutions for compressible scatterers, the model is initially implemented with both the particle and the surrounding fluid as fluid domains supporting linear, scalar acoustic representations. In Sec. IV C elastic scatterers are considered; the particle is represented as a linear elastic solid, and the effect of shear wave propagation on the resulting forces is discussed.

The model is axi-symmetric, with the axis of symmetry on the left-hand boundary of Fig. 1. The particle is represented by a semi-circular domain, with a base on this axis, and the surrounding fluid is shown as a rectangular domain, also with a base on this axis. Non-reflecting boundary conditions (NRBCs) delimit the computational domain and allow an acoustic field to be introduced by specifying pressure conditions over the boundary while simultaneously absorbing the majority of acoustic energy incident upon the boundary from the scatterer. COMSOL provides a NRBC based on 
TABLE I. Baseline parameters.

\begin{tabular}{ll}
\hline \hline Parameter & \multicolumn{1}{c}{ Value } \\
\hline Particle speed of sound, $c_{p}$ & $6559 \mathrm{~m} / \mathrm{s}$ \\
Particle density, $\rho_{p}$ & $2000 \mathrm{~kg} / \mathrm{m}^{3}$ \\
Particle radius & $10 \mu \mathrm{m}$ \\
Mesh size & $0.2 \mu \mathrm{m}$ (particle boundary) \\
& $4 \mu \mathrm{m}$ (maximum) \\
Distance of Particle from & $3 \lambda / 8$ (force is maximum here \\
pressure node & for small particles) \\
Fluid speed of sound, $c_{f}$ & $1480 \mathrm{~m} / \mathrm{s}$ \\
Fluid density, $\rho_{f}$ & $1000 \mathrm{~kg} / \mathrm{m}^{3}$ \\
Fluid Domain dimensions & $140 \mu \mathrm{m} \mathrm{high} \mathrm{(along} \mathrm{acoustic} \mathrm{axis)}$ \\
& $70 \mu \mathrm{m} \mathrm{wide}$ \\
Frequency & $1 \mathrm{MHz}$ \\
Wavelength in fluid, $\lambda$ & $1.48 \mathrm{~mm}$ \\
\hline \hline
\end{tabular}

Bayliss et $a l .{ }^{40}$ In this manner the simulated domain spans only a fraction of a wavelength. Bayliss et al. also suggest that these second-order boundary conditions work acceptably even in the near field and this is borne out by results below. It has also been verified that similar numerical accuracy can be produced using a perfectly matched layer (PML) in place of the NRBC for the results presented in Sec. IV B. The particle and fluid domains are coupled such that only normal velocity components are transmitted across the boundary. The model is solved using a harmonic analysis at a frequency of interest. The forces are evaluated by performing the integration from Eq. (5) over the boundary of the particle.

Figure 1 also shows the pressure distribution of the scattered field resulting from a sphere when a standing wave of pressure amplitude of $200 \mathrm{kPa}$ is applied via the boundary. The material and domain parameters are listed in Table I; these parameters will be used throughout the paper as a baseline set of data unless otherwise stated. Evaluating Eq. (5) over the particle surface, we deduce a radiation force of $127.66 \mathrm{pN}$ acting upwards on the particle, which compares well with the analytical result of $127.83 \mathrm{pN}$ predicted by Yosioka and Kawasima. It is interesting to note that in the case of a fixed, rigid sphere (i.e., without the momentum flux correction required for a moving sphere), the force is $150.80 \mathrm{pN}$.

\section{Mesh and domain dependency, computational load}

To evaluate the mesh density and fluid domain size required to achieve an appropriate level of accuracy, these parameters were varied using the parameters in Table I as a basis. By fluid domain size, we mean the length of the rectangular boundary that delimits the fluid region. We also investigated using a circular fluid domain and found the difference in the results negligible. Figure 2 and Fig. 3 show how the predicted force compares to Hasegawa's analytical results for the force on a compressible, fluid sphere ${ }^{26,41}$ (see Sec. IV B, below). Both a uniform mesh and a mesh that is denser at the particle boundary were tested.

We also compare in Fig. 2 the effect of making the small scattering approximation (see above), decomposing

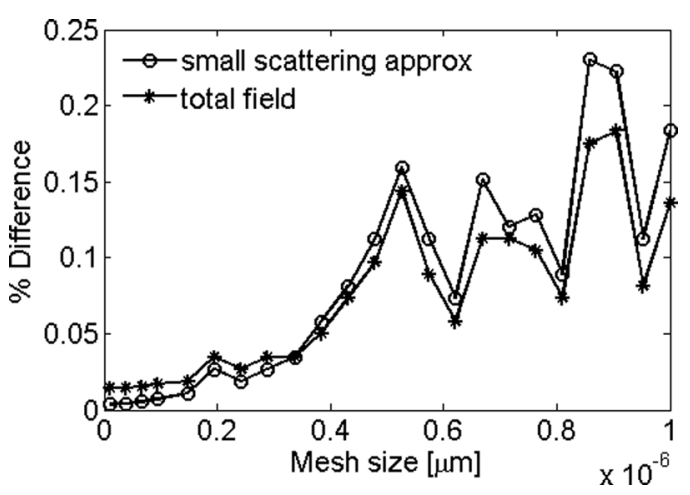

FIG. 2. Mesh dependency plot with y-axis as the \% difference from Hasegawa analytical result with a domain size of $70 \mu \mathrm{m}$.

the field into incident and scattered components. The results show higher accuracy (approaching $0.005 \%$ difference from Hasegawa's analytical result in the limit of mesh refinement) when making the small scatterer approximation (see above for why this may be the case) compared to the full-field implementation (approaching $0.015 \%$ in the limit of mesh refinement). Both results will be satisfactory for most applications. If the model were modified to predict forces in progressive waves the difference is likely to become more important, as the resulting forces are much smaller, and more likely to be obscured by these numerical errors. It will be demonstrated below (Sec. IV B) that for larger particles, the error introduced by considering only the incoming/scattered mixed products becomes significant.

At these settings running a set of 100 simulations (to lessen the effects of setup overheads on the simulation time) takes a total of $186 \mathrm{~s}$ (solving for 13913 degrees of freedom on a desktop PC with a $2.67 \mathrm{GHz}$ Intel(R) Xeon(R) CPU with 12.0 GB RAM). This demonstrates the advantage of the FEA perturbation approach over the lengthier Navier-Stokes simulations described by Wang and Dual ${ }^{23}$ in which an individual simulation takes many hours to complete. It should be noted, however, that the Wang and Dual approach also models viscous effects and the resulting streaming fields. It has been shown by Doinikov ${ }^{16}$ that for a spherical particle whose diameter is significantly greater than the thermal and viscous penetration depths (of order $<1 \mu \mathrm{m}$ in the cases examined in

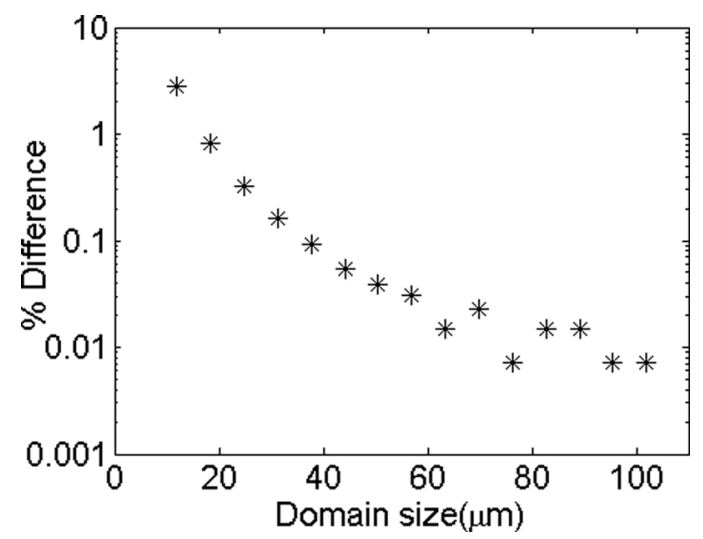

FIG. 3. Domain size variation plot of fluid model with y-axis as the $\%$ difference from Hasegawa analytical result with a mesh size of $0.2 \mu \mathrm{m}$. 
TABLE II. Forces on a rigid cylinder: FEA perturbation compared to Wang and Dual results. Parameters are normalized as in Wang and Dual (Ref. 23) and Haydock (Ref. 21).

\begin{tabular}{lcccc}
\hline \hline Wang and Dual & $\begin{array}{c}\text { Percent of difference } \\
\text { (Wang and Dual } \\
\text { analytical }\left(10^{-5}\right)\end{array}$ & $\begin{array}{c}\text { FEA perturbation } \\
\left(10^{-5}\right)\end{array}$ & $\begin{array}{c}\text { Percent of difference } \\
\text { (Wang and Dual anatyical / } \\
\text { FEA perturbation) }\end{array}$ \\
\hline 5 & -1.2337 & -0.813 & -1.2353 & -0.130 \\
10 & -4.932 & -0.000 & -4.9521 & -0.408 \\
20 & -19.654 & -0.051 & -19.718 & -0.326 \\
40 & -76.719 & -0.508 & -76.774 & -0.072 \\
80 & -257.22 & 1.571 & -249.82 & 2.877 \\
\hline \hline
\end{tabular}

this paper), the viscous and thermal effects can be ignored to a reasonable degree of accuracy.

\section{RESULTS}

\section{A. Force on a rigid cylinder in a standing wave}

Wang and Dual ${ }^{23}$ present both an analytical solution and a finite volume (FVM) approach that predict the force on a rigid cylinder in a standing wave. In order to verify the analytical result, and evaluate the efficiency of the FEA perturbation method, this geometry is explored first. Table II compares results of both their analytical and FVM models to our perturbation FEA approach with the particle implemented as a linear elastic solid. The set of parameters used are as described in their Table II. Readers are referred to Wang and Dual ${ }^{23}$ for these normalized parameters. The analytical results below are calculated from their equations, and the remaining data are taken from their Table II. For these results, the model described above was modified to no longer be axially symmetric and can be seen in Fig. 4. The domain size was of the same size as that used by Wang and Dual.

We see that the FEA perturbation approach provides good results with similar agreement to the analytical result as their FVM Navier-stokes method. Wang and Dual also calculate results for systems where viscosity is important (small radius particle or high viscosity); however, our approach is not valid for this.

\section{B. Force on a fluid sphere in a standing wave}

In this section we initially verify the FEA perturbation method for small, compressible spheres as first derived analytically by Yosioka and Kawasima (and Gor'kov for arbitrary standing wave fields) and then test the small scatterer

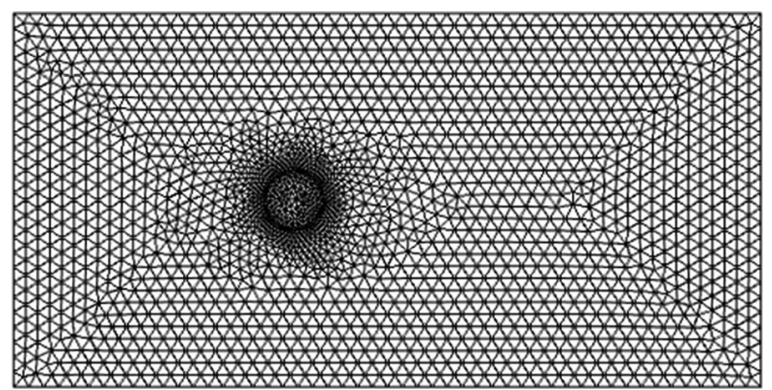

FIG. 4. Finite element model for a rigid cylinder in a standing wave. All external boundaries are NRBCs. assumption against Hasegawa's results for arbitrary sized spheres. Hasegawa presents the more general case of an elastic sphere, and the fluid sphere can be considered as the special case of an elastic sphere with zero shear velocity. However, to implement the fluid particle case in FEA with COMSOL it is necessary to use acoustic elements (i.e., a potential formulation) for the particle, as solid elements with zero shear velocity are not supported. See the next section for discussion of Hasegawa's equations.

Table III shows results for the parameters described in Table I but over a range of particle sizes. For the smaller radii, good agreement is found; however, when the radius reaches $80 \mu \mathrm{m}$ ( $k a$ of 0.34 ), the difference is greater than $7 \%$ due to the error in making the small scatterer approximation described above. The Hasegawa results, from Eq. (10) below, are seen to match the results more closely. For experimental work it is useful to see in more detail how the force deviates from the small radius approximation of Gor'kov or Yosioka and Kawasima as the radius increases (Fig. 5). Throughout the range considered, the FEA perturbation approach follows the Hasegawa results closely. See Sec. III B above for explanation of the difference between the FEA perturbation results labeled "mixed terms only," and "total."

Figure 6 shows how the direction of the radiation force on a compressible sphere in a plane standing wave, as predicted by Gor'kov in Eq. (1) above, is affected by the compressibility and density of the particle. The line of zero force is of interest as near this line small numerical offset errors lead to higher percentage errors.

In order to verify the FEA result over a range of parameters, the radiation force was calculated on an array of particle material properties (other properties as Table I) covering the range of compressibilities $\left(4.56 \mathrm{e}-11\right.$ to $6.85 \mathrm{e}-10 \mathrm{~Pa}^{-1}$ in 13 steps) and densities (100 to $2500 \mathrm{~kg} \mathrm{~m}^{-3}$ in 12 steps) such that all 156 combinations of these properties are explored (but ignoring the 18 cases where the force is less than $15 \mathrm{pN}$ ), it is found that the FEA perturbation result is always within $0.25 \%$ of the Hasegawa result.

\section{Three-dimensional FEA perturbation model of a fluid sphere}

The FEA perturbation approach is also computationally suited to 3D particle modeling. The model has been implemented in 3D, and for the parameters in Table I (but with an increase in mesh size to $0.4 \mu \mathrm{m}$ ) produces a prediction for 
TABLE III. Comparison of FEA perturbation result with analytical results for forces on compressible spheres. All forces in pN.

\begin{tabular}{lcccc}
\hline \hline $\begin{array}{l}\text { Particle } \\
\text { Radius }(\mu \mathrm{m})\end{array}$ & Force Y\&K & $\begin{array}{c}\text { Force Haseqawa } \\
\text { (fluid particle) }\end{array}$ & $\begin{array}{c}\text { Force FEA } \\
\text { perturbation }\end{array}$ & $\begin{array}{c}\text { Percent of } \\
\text { difference } \\
\text { difference } \\
\text { (Y\&K/FEA) }\end{array}$ \\
\hline 5 & 15.979 & 15.975 & 15.966 & 0.062 \\
10 & 127.83 & 127.68 & 127.645 & 0.137 \\
20 & 1022.7 & 1017.9 & 1018.7 & 0.433 \\
40 & 8181.2 & 8031.6 & 8033.0 & 0.029 \\
80 & 65450 & 60824 & 60682 & -0.076 \\
\hline \hline
\end{tabular}

the force that is within $0.03 \%$ of the analytical predictions of Hasegawa above. This is comparable to the accuracy of the 2D model. The model was found to produce solutions on an Intel Nehalem compute node with 22 GB RAM in approximately $320 \mathrm{~s}$. A 3D model will be used in the future to investigate forces on particles that are not rotationally symmetric. With the 3D model it will also be possible to calculate torques on particles set at an angle to the direction of sound propagation, and model multi-particle and particle-wall interactional effects.

\section{Force on an elastic sphere of arbitrary size in a standing wave}

Hasegawa presents an analytical solution for the radiation force on elastic spheres of arbitrary size. ${ }^{26,41}$ In order to normalize results, he uses a radiation force function, $Y_{s t}$, defined as the force per unit cross section of a sphere and unit energy density of the standing wave field. Mitri ${ }^{42}$ points out two typographical errors in Hasegawa.

(a) Equation (19) of Hasegawa: ${ }^{26}$

The final expression for the radiation force function for a standing wave field should be

$Y_{s t}=\frac{8}{x^{2}} \sum_{n=0}^{\infty}(n+1)(-1)^{n+1}\left[\beta_{n}\left(1+2 \alpha_{n+1}\right)-\beta_{n+1}\left(1+2 \alpha_{n}\right)\right]$

without the additional $x^{2}$ term found in the original.

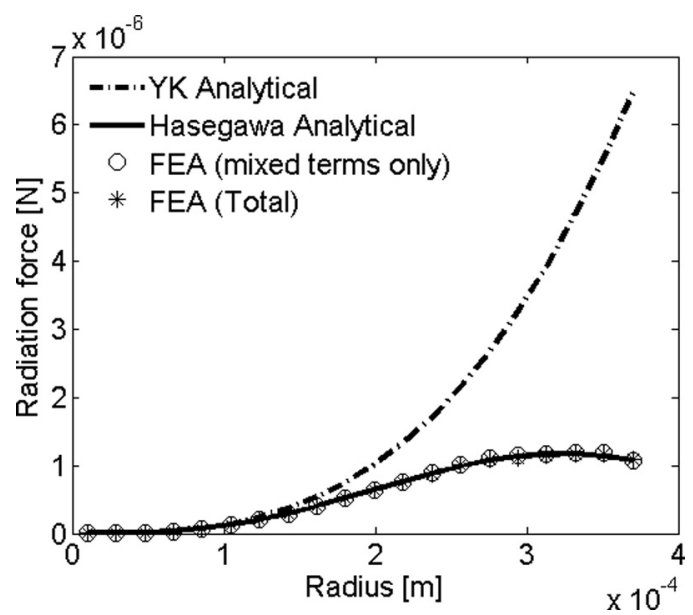

FIG. 5. Force on a compressible sphere as the radius is increased. See Table I for parameters. (b) Equation (9) of Hasegawa: ${ }^{41}$

The equation for the coefficient term, $B_{n}$, should be

$B_{n}=\frac{2 n(n+1) j_{n}\left(x_{2}\right)}{\left(2 n^{2}-x_{2}^{2}-2\right) j_{n}\left(x_{2}\right)+2 x_{2} j_{n+1}\left(x_{2}\right)}$,

where previously there was a squaring of the second $x_{2}$ in the denominator.

We further notice another typographical error.

(c) Equation (5) of Hasegawa: ${ }^{41}$

The function, $F_{n}$, is given as

$F_{n}=\frac{\rho x^{*}\left[n j_{n}\left(x^{*}\right)-x^{*} j_{n+1}\left(x^{*}\right)\right]}{\rho^{*} j_{n}\left(x^{*}\right)}$

but should be

$F_{n}=\frac{\rho\left[n j_{n}\left(x^{*}\right)-x^{*} j_{n+1}\left(x^{*}\right)\right]}{\rho^{*} j_{n}\left(x^{*}\right)}$.

This can be seen most directly by allowing the shear velocity to approach zero in Hasegawa's elastic equation [Eq. (7) in Ref. 41]. Please refer to the original papers for the definition and meaning of the symbols used.

We can now mutually verify these corrected Hasegawa equations against the FEA perturbation method. Representing the particle using linear elastic elements in COMSOL, the following results are obtained.

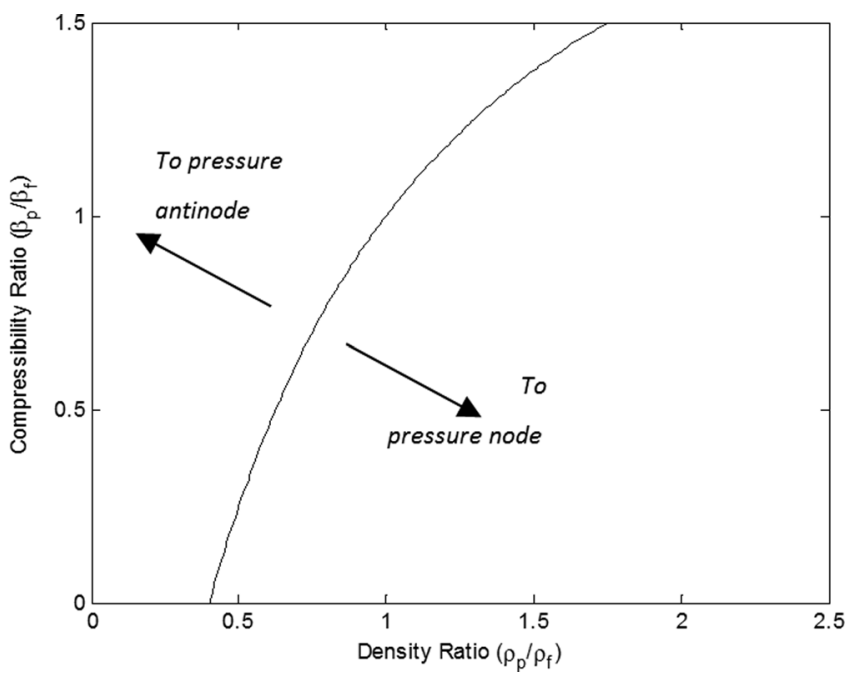

FIG. 6. Particle-fluid combinations for which the force on a small particle in a plane standing wave equals zero. Particles to the right of and below the line move to the pressure node in a plane standing wave. 


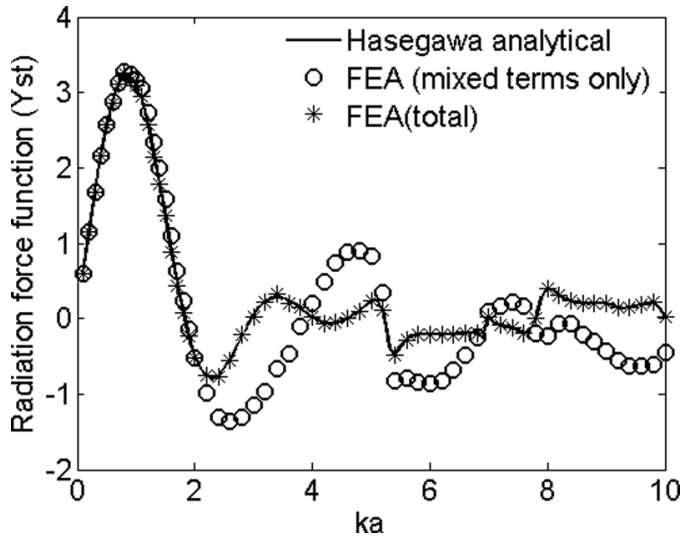

FIG. 7. Force function for a stainless steel sphere vs $k a$ (where $k$ is the wave number and $a$ is the particle radius), compared to model results.

Figure 7 plots the force on a steel sphere as the radius is increased. The example is taken from Hasegawa, ${ }^{26}$ and the sphere has a density of $7900 \mathrm{~kg} \mathrm{~m}^{-3}$, longitudinal velocity of $5240 \mathrm{~m} \mathrm{~s}^{-1}$, and shear velocity of $2978 \mathrm{~m} \mathrm{~s}^{-1}$. The results show an identical shape to that plotted in his Fig. 2, indicating that the errors pointed out above are typographic, since the corrections are required to reproduce the original figure.

It can be seen that after an initial growth, the force oscillates about zero, in a complex manner. Our FEA perturbation results are seen to be in close agreement with Hasegawa's. Also plotted is the force calculated using the small scattering approximation that omits all but the incoming/scattered mixed products (see above), and it is interesting to note how this provides a close approximation until $k a \approx 2$ and then begins to fail as the particle size grows.

Table IV is also presented to highlight results that are of experimental significance. It shows the modeled force on particles made from a number of different materials and compares the result to the Yosioka and Kawasima approximation. It can be seen that for some materials there is a significant difference-particularly for the polystyrene beads.

\section{E. The force on compressible spheroids in a standing wave}

The cases discussed above and indeed the majority of cases modeled in the literature are based on scatterers of simple geometry such as spheres and cylinders. While this is necessary to provide useful analytical expressions for scattered fields, a significant advantage of a numerical approach such as the FEA perturbation method discussed here is the ability to model the force on arbitrary geometries. This is of importance as many cells (including the commonly encountered red blood cells and neuronal cells) are known to have geometries that are neither cylindrical nor spherical. We begin by verifying our model against the results presented by Marston et $a l^{24}$ for the radiation force on rigid, fixed spheroids. Although our ultimate aim is to model arbitrary shaped scatters of an elastic nature, Marston et al. do not extend their theory to include elasticity therefore verification is limited to the arbitrary shape aspect, and as such we use fluid elements. They define a shape parameter,

$$
\varepsilon=[(b / a)-1]
$$

where $a$ and $b$ are the radii in the direction of, and perpendicular to, sound propagation, respectively.

They derive an approximate expression for the ratio between the force on the spheroid and a sphere of identical volume that is valid for $|\varepsilon| \ll 1$,

$$
\frac{F_{\text {spheroid }}(\varepsilon)}{F_{\text {sphere }}} \approx 1+\left(\frac{6}{25}\right) \varepsilon+\left(\frac{9}{875}\right) \varepsilon^{2}+\cdots .
$$

Figure 8 compares Marston's analytical result and our FEA perturbation model. It can be seen that there is good agreement between the two, even approaching $\varepsilon=1$. The modeled parameters for the simulation are as described in Table I except the particle boundary is fixed, removing any dependence on particle properties.

Relaxing the constraints of having a rigid, fixed particle and small $\varepsilon$, Fig. 9 shows how the force on compressible particles (of constant resting volume) changes as they are deformed for a variety of particle densities. The variation, however, is also sensitive to the particle's compressibility. Figure 10 shows how the force varies for a range of both densities and compressibilities; this is for an oblate spheroid with $\varepsilon=2.41$. Areas of low force with correspondingly higher numerical errors have been excluded and so left white.

It can be seen that when the particle is of the same density as the surrounding fluid, the ratio is close to unity, and the shape makes little difference. This result is of particular importance in cell manipulation and sorting applicationsthe near neutral buoyancy of most cells means that small deformations away from a spherical shape are unlikely to have a significant effect on the magnitude of the force

TABLE IV. Effect of elastic material properties on modeled force compared to Yosioka and Kawasima (Y\&K), and Hasegawa. Other parameters as listed in

\begin{tabular}{|c|c|c|c|c|c|c|c|c|}
\hline & $\begin{array}{l}\text { Longitudinal } \\
\text { velocity } \\
(\mathrm{m} / \mathrm{s})\end{array}$ & $\begin{array}{l}\text { Shear } \\
\text { velocity } \\
(\mathrm{m} / \mathrm{s})\end{array}$ & $\begin{array}{l}\text { Density } \\
\left(\mathrm{kg} / \mathrm{m}^{3}\right)\end{array}$ & $\begin{array}{l}\text { FEA modeled } \\
\text { force }(\mathrm{N})\end{array}$ & $\begin{array}{c}\mathrm{Y} \& \mathrm{~K} \\
\text { force }(\mathrm{N})\end{array}$ & $\begin{array}{l}\text { Percent of } \\
\text { difference } \\
\text { Y\&K/FEA }\end{array}$ & $\begin{array}{l}\text { Hasegawa } \\
\text { force }(\mathrm{N})\end{array}$ & $\begin{array}{c}\text { Percent of } \\
\text { difference } \\
\text { Hasegawa/FEA }\end{array}$ \\
\hline Glass (crown) & 5100 & 2800 & 2240 & $1.31 \mathrm{E}-10$ & $1.33 \mathrm{E}-10$ & 1.70 & $1.310 \mathrm{E}-10$ & 0.040 \\
\hline Nylon, 6/6 & 2600 & 1100 & 1120 & $5.93 \mathrm{E}-11$ & $6.67 \mathrm{E}-11$ & 11.2 & $5.926 \mathrm{E}-11$ & 0.018 \\
\hline Polystyrene, Styron 666 & 2400 & 1150 & 1050 & $4.27 \mathrm{E}-11$ & $5.57 \mathrm{E}-11$ & 23.4 & $4.266 \mathrm{E}-11$ & 0.007 \\
\hline Steel-stainless 347 & 5790 & 3100 & 7890 & $1.80 \mathrm{E}-10$ & $1.81 \mathrm{E}-10$ & 0.40 & $1.798 \mathrm{E}-10$ & 0.037 \\
\hline Aluminum-rolled & 6420 & 3040 & 2700 & $1.43 \mathrm{E}-10$ & $1.44 \mathrm{E}-10$ & 0.61 & $1.434 \mathrm{E}-10$ & 0.021 \\
\hline
\end{tabular}
Table I. 


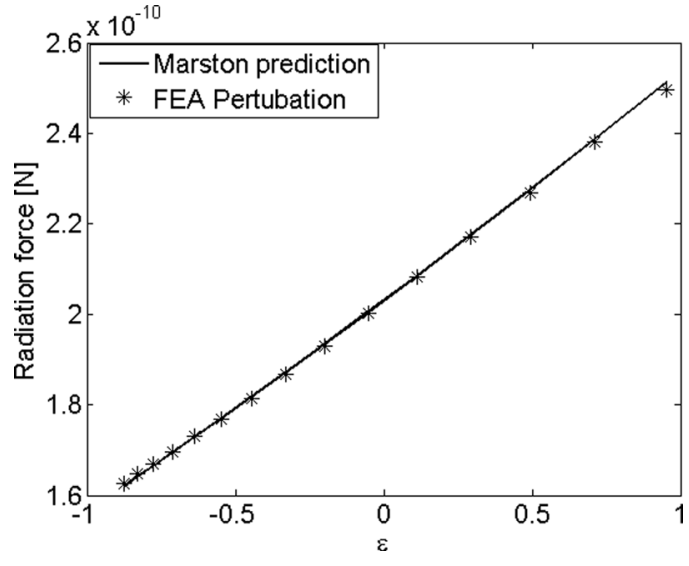

FIG. 8. Force on a fixed, rigid spheroid over a range of deformations. FEA perturbation result compared to analytical result. The force is in the same direction as that on a sphere: towards the nearest pressure node.

experienced. However, non-spherical cells such as red blood cells have been shown to experience a torque that tends to align them with the pressure node of a system, ${ }^{43}$ so there may still be scope to exploit this for cell sorting on a shape basis.

\section{CONCLUSIONS}

This paper has presented and verified a numerical method for predicting the acoustic radiation force on particles of arbitrary shape, composition, and size (in the nonviscous approximation). This paves the way for modeling more practically relevant particles such as biological cells, and the interactions of multi-body systems. This has potential to facilitate applications such as cell sorting and manipulation. The method has been verified against a range of analytical solutions and shown to produce accurate results in a short computational time, and we have highlighted a number of interesting trends relating to particle shape, size, and elasticity.

Future work will investigate complex, multi-material particles. We will also pursue 3D models to better predict multi-body interactions and torques.

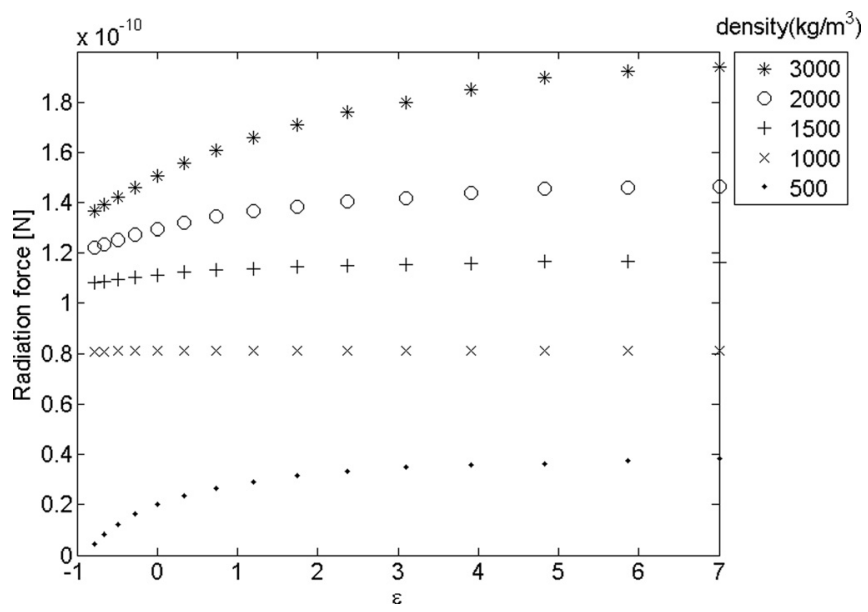

FIG. 9. Force on a spheroid of constant volume over a range of deformations and particle densities.

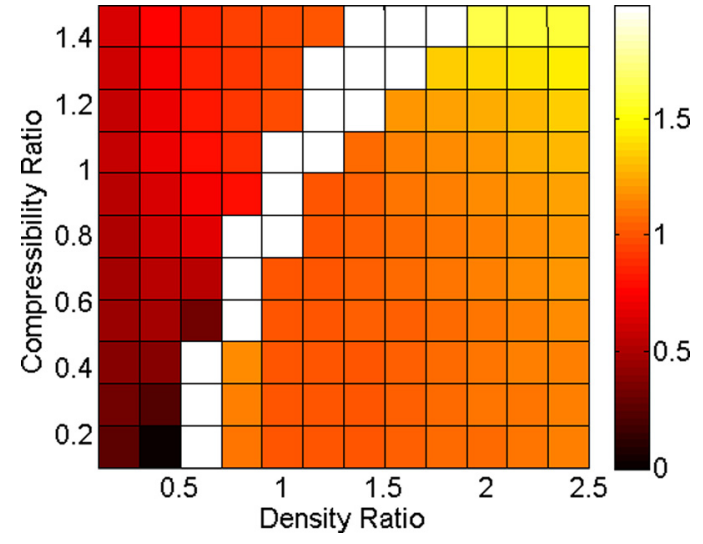

FIG. 10. (Color online) Fspheroid/Fsphere, for a range of particle properties, with a spheroid of $\varepsilon=-2.41$ compared to a sphere of the same volume (excluding region $F$ sphere $<15 \mathrm{pN}$ ).

\section{ACKNOWLEDGMENTS}

This work has been funded by the Engineering and Physical Sciences Research Council, UK through the Sonotweezers project (Grant EP/G012075). The authors thank the Sonotweezers project partners at the Universities of Bristol, Dundee, and Glasgow for their support and assistance in this research.

${ }^{1}$ D. Bazou, W. T. Coakley, A. J. Hayes, and S. K. Jackson, "Long-term viability and proliferation of alginate-encapsulated 3-D HepG2 aggregates formed in an ultrasound trap," Toxicol. In Vitro 22, 1321-1331 (2008).

${ }^{2}$ J. Hultström, O. Manneberg, K. Dopf, H. M. Hertz, H. Brismar, and M. Wiklund, "Proliferation and viability of adherent cells manipulated by standing-wave ultrasound in a microfluidic chip" Ultrasound Med. Biol. 33, 175-181 (2006).

${ }^{3}$ J. Svennebring, O. Manneberg, P. Skafte-Pedersen, H. Bruus, and M. Wiklund, "Selective bioparticle retention and characterization in a chipintegrated confocal ultrasonic cavity," Biotechnol. Bioeng. 103, 323-328 (2009).

${ }^{4}$ P. Glynne-Jones, R. J. Boltryk, M. Hill, F. Zhang, L. Dong, J. S. Wilkinson, T. Brown, T. Melvin, and N. R. Harris, "Multi-modal particle manipulator to enhance bead-based bioassays," Ultrasonics 50, 235-239 (2010).

${ }^{5}$ C. Ratier and M. Hoyos, "Acoustic programming in step-split-flow lateraltransport thin fractionation," Anal. Chem. 82, 1318-1325 (2010).

${ }^{6}$ P. Augustsson, L. B. Aberg, A. M. K. Sward-Nilsson, and T. Laurell, "Buffer medium exchange in continuous cell and particle streams using ultrasonic standing wave focusing," Microchim. Acta 164, 269-277 (2009). ${ }^{7}$ K. A. Garvin, D. C. Hocking, and D. Dalecki, "Controlling the spatial organization of cells and extracellular matrix proteins in engineered tissues using ultrasound standing wave fields," Ultrasound Med. Biol. 36, 1919-1932 (2010).

${ }^{8}$ L. V. King, "On the acoustic radiation pressure on spheres," Proc R. Soc. London Ser. A 147, 212-240 (1934).

${ }^{9} \mathrm{~K}$. Yosioka and Y. Kawasima, "Acoustic radiation pressure on a compressible sphere," Acustica 5, 167-173 (1955).

${ }^{10}$ L. P. Gor'kov, "On the forces acting on a small particle in an acoustical field in an ideal fluid," Sov. Phys. Dokl. 6, 773-775 (1962).

${ }^{11}$ T. F. W. Embleton, "Mean force on a sphere in a spherical sound field. I. Theoretical," J. Acoust. Soc. Am. 26, 40-45 (1954).

${ }^{12}$ X. C. Chen and R. E. Apfel, "Radiation force on a spherical object in the field of a focused cylindrical transducer," J. Acoust. Soc. Am. 101, 2443-2447 (1997).

${ }^{13}$ M. Barmatz and P. Collas, "Acoustic radiation potential on a sphere in plane, cylindrical, and spherical standing wave fields," J. Acoust. Soc. Am. 77, 928-945 (1985).

${ }^{14} \mathrm{P}$. J. Westervelt, "The theory of steady forces caused by sound waves," J. Acoust. Soc. Am. 23, 312-315 (1951). 
${ }^{15}$ A. A. Doinikov, "On the radiation pressure on small spheres," J. Acoust. Soc. Am. 100, 1231-1233 (1996).

${ }^{16}$ A. A. Doinikov, "Acoustic radiation force on a spherical particle in a viscous heat-conducting fluid. 1. General formula," J. Acoust. Soc. Am. 101, 713-721 (1997).

${ }^{17}$ L. V. King, "On the acoustic radiation pressure on circular discs: Inertia and diffraction corrections," Proc. R. Soc. London Ser. A 153, 1-16 (1935).

${ }^{18}$ W. J. Xie and B. Wei, "Dynamics of acoustically levitated disk samples," Phys. Rev. E 70, 046611 (2004).

${ }^{19}$ W. T. Shi and R. E. Apfel, "Deformation and position of acoustically levitated liquid drops," J. Acoust. Soc. Am. 99, 1977-1984 (1996).

${ }^{20}$ F. Cai, L. Meng, C. Jiang, Y. Pan, and H. Zheng, "Computation of the acoustic radiation force using the finite-difference time-domain method," J. Acoust. Soc. Am. 128, 1617-1622 (2010).

${ }^{21}$ D. Haydock, "Lattice Boltzmann simulations of the time-averaged forces on a cylinder in a sound field," J. Phys. A 38, 3265-3277 (2005).

${ }^{22}$ F. G. Mitri, "Theoretical calculation of the modulated acoustic radiation force on spheres and cylinders in a standing plane wave-field," Physica D 212, 66-81 (2005).

${ }^{23}$ J. T. Wang and J. Dual, "Numerical simulations for the time-averaged acoustic forces acting on rigid cylinders in ideal and viscous fluids," J. Phys. A 42285502 (2009).

${ }^{24}$ P. L. Marston, W. Wei, and D. B. Thiessen, in Innovations in Nonlinear Acoustics, edited by A. A. Atchley, V. W. Sparrow, and R. M. Keolian (AIP, Melville, NY, 2006), Vol. 838, pp. 495-499.

${ }^{25}$ T. Hasegawa and K. Yosioka, "Acoustic radiation force on a solid elastic sphere," J. Acoust. Soc. Am. 46, 1139-1143 (1969).

${ }^{26}$ T. Hasegawa, "Acoustic radiation force on a sphere in a quasistationary wave field-theory," J. Acoust. Soc. Am. 65, 32-40 (1979).

${ }^{27}$ M. Gröschl, "Ultrasonic separation of suspended particles-Part I: Fundamentals," Acustica 84, 432-447 (1998).

${ }^{28}$ H. Nowotny, E. Benes, and M. Schmid, "Layered piezoelectric resonators with an arbitrary number of electrodes (general one-dimensional treatment)," J. Acoust. Soc. Am. 90, 1238-1245 (1991).

${ }^{29}$ M. Hill, Y. Shen, and J. J. Hawkes, "Modelling of layered resonators for ultrasonic separation," Ultrasonics 40, 385-392 (2002).
${ }^{30}$ M. Hill, R. J. Townsend, and N. R. Harris, "Modelling for the robust design of layered resonators for ultrasonic particle manipulation," Ultrasonics 48, 521-528 (2008).

${ }^{31}$ R. J. Townsend, M. Hill, N. R. Harris, and N. M. White, "Modelling of particle paths passing through an ultrasonic standing wave," Ultrasonics 42, 319-324 (2004).

${ }^{32}$ A. Neild, S. Oberti, A. Haake, and J. Dual, "Finite element modeling of a microparticle manipulator," Ultrasonics 44, e455-e460 (2006).

${ }^{33}$ R. J. Townsend, M. Hill, N. R. Harris, and N. M. White, "Investigation of two-dimensional acoustic resonant modes in a particle separator," Ultrasonics 44, e467-e471 (2006).

${ }^{34}$ S. M. Hagsater, A. Lenshof, P. Skafte-Pedersen, J. P. Kutter, T. Laurell, and $\mathrm{H}$. Bruus, "Acoustic resonances in straight micro channels: Beyond the 1D-approximation," Lab Chip 8, 1178-1184 (2008).

${ }^{35}$ K. A. Fisher and R. Miles, "Modeling the acoustic radiation force in microfluidic chambers," J. Acoust. Soc. Am. 123, 1862-1865 (2008).

${ }^{36}$ S.-T. Kang and C.-K. Yeh, "Potential-well model in acoustic tweezers," IEEE Trans. Ultrason. Ferroelectr. Frequency Control, 57, 1451-1459 (2010).

${ }^{37}$ Y. Y. Liu, J. H. Hu, and C. S. Zhao, "Dependence of acoustic trapping capability on the orientation and shape of particles," IEEE Trans. Ultrason. Ferroelectr. Frequency Control 57, 1443-1450 (2010).

${ }^{38}$ H. Bruus, Theoretical Microfluidics (Oxford University Press, New York, 2008).

${ }^{39}$ M. J. Lighthill, Waves in Fluids (Cambridge University Press, New York, 2001).

${ }^{40}$ A. Bayliss, M. Gunzburger, and E. Turkel, "Boundary-conditions for the numerical-solution of elliptic-equations in exterior regions," SIAM J. Appl. Math. 42, 430-451 (1982).

${ }^{41}$ T. Hasegawa, "Comparison of two solutions for acoustic radiation pressure on a sphere," J. Acoust. Soc. Am. 61, 1445-1448 (1977).

${ }^{42}$ F. G. Mitri, "Acoustic radiation force acting on elastic and viscoelastic spherical shells placed in a plane standing wave field," Ultrasonics 43 , 681-691 (2005)

${ }^{43}$ W. T. Coakley, D. Bazou, J. Morgan, G. A. Foster, C. W. Archer, K. Powell, K. A. J. Borthwick, C. Twomey, and J. Bishop, "Cell-cell contact and membrane spreading in an ultrasound trap," Colloid Surf. B 34, 221-230 (2004) 